\title{
QUALITY OF LIFE AFTER BARIATRIC SURGERY
}

Cuenca RM. Quality of life after bariatric surgery. Arq Gastroenterol. 2014;51(3):163-4.

HEADINGS - Bariatric surgery. Quality of life.

Quality of life after bariatric surgery can be investigated in two different ways. On one hand, it can be assessed in terms of improvements in the patient's overall physical health. There is ample empirical and statistically supported evidence in the literature showing improvements in comorbidities after bariatric surgery. Data shows that surgical intervention in the treatment of obesity not only improves the symptoms of the disorder but it can also cure it, often times extending patients lives by helping them lose weight. The data provided in the literature supporting evidence of improvement in the patient's life quality post-surgery are often quantitative, easily measured, data. However, in the fourteen years of experience that my multidisciplinary team and I have with bariatric surgery, we have come to observe that while patients do value the overall positive impact that the procedure has on their "physical health", they measure the overall impact of the procedure on their quality of life on different scales, often based on their personal expectations as to the outcome of the surgery. Some patients believe that the weight loss achieved as a consequence of the procedure will help them get better jobs or a promotion, or that they will be able to choose to have a career in a field that was completely unavailable to them because of their obesity. Other patients think their lives will improve because they will be able to practice sports, to take public transportation, to fit into a pair of jeans; while others think that losing weight is the only way they will be accepted by society or the only way they will ever find true love. Given this myriad of "definitions" that patients may have for quality of life, how can doctors measure the extent to which bariatric surgery improves the quality of life of their patients beyond improvements in health parameters?

Several factors have contributed to the success of bariatric surgery in the treatment of obesity. The support of a multidisciplinary team pre- and post-surgery and technological advances have certainly had a major role not only in the patient's own assessment of their quality of life, but also in reducing the procedure's morbimortality, the length of the procedure, the amount of time patients need to stay in the hospital (around 48 hours now), and the number of patients that need to be treated in an intensive care unit (a rare occurrence today). Altogether these factors improve patients' quality of life. For example, many patients who seek intervention are independent professionals who must work to make a living and support themselves and their families. Therefore, the amount of time they had to take off work to undergo the procedure used to be a great concern. As a result of the new advances in bariatric surgery, patients are able to resume their normal daily activities in just a few days. This eliminates this parameter as a determining factor in patients' decision to undergo the surgery making the procedure more accessible to them.

Aesthetics is usually a point of contention in discussions of patients' motives to seek bariatric surgery. Many professionals believe that aesthetics should not drive the decision to undergo surgery. We do not address such cases here. We will not discuss patients that want to have bariatric surgery to "look good". Our focus is on those patients that meet all the legal and medical criteria required to qualify for surgical treatment. Aesthetics, however, can be used as a measure of improvement of quality of life. Even when patients' ultimate goal is not to "look good", one cannot ignore their reports on how immensely happy they feel when, after reaching their ideal weight post surgical treatment, they are actually able to shop for clothing at a regular store or to go to public places without feeling embarrassed. Some patients comment on how much their sexual life improved as well, and some end up participating in high-level sports, a few becoming marathoners after the treatment. These are all examples of the positive impact of bariatric surgery in the patients' quality of life.

\footnotetext{
The author indicated no potential conflicts of interest.

* Professor Adjunto da Universidade de Brasília, DF, Brasil. Membro Titular do Colégio Brasileiro de Cirurgia Digestiva.
} 
While there are many benefits to bariatric surgical intervention, there can also be downsides to the treatment. For example, patients for whom aesthetics is important often fail to realize that the desired weight loss will lead to an increase in excess of skin or surgical scars and they become frustrated when faced with that reality. There are also prospective patients that see bariatric surgery as a magical tool that will help them lose all their weight overnight with minimum personal effort. These are individuals that have heard the success stories but that often do not understand that the actual surgical procedure is only one step in a long treatment that requires serious patient commitment and effort. The treatment can significantly improve patients' quality of life, but to reach this outcome patients must change their lifestyle. For example, they need to change their eating habits and adopt a daily exercise routine, never an easy task. The role of multidisciplinary teams becomes even prominent here as one of the key factors in the success of bariatric surgery. It is their responsibility to educate prospective patients and to make them aware that while a solution to their comorbidities and quality of life improvement are within their reach, this will only come to happen if they comply with the entire obesity treatment. The team must conduct thorough interviews with the patients to learn what quality of life means to them and determine what improvements they are expecting to achieve with the surgical treatment. This way, we can more efficiently manage expectations and prevent disappointment and frustration. Patients should know what outcomes they should expect but by the end treatment but, most importantly, which ones they should not expect. Disappointment with surgery outcomes may lead to depression which may lead patients to the very behavior that pushed them to become obese in the first place, often compulsive eating. In fact, disappointment and frustration with the treatment may even lead to a gamut of other problems that were absent prior to the obesity treatment (e.g., alcoholism, violence, kleptomania, etc.). False expectations can severely hinder the treatment of morbid obesity, thus they must be addressed effectively and efficiently both pre- and post-surgical intervention.

In summary, there is ample evidence that the surgical treatment of obesity can positively impact patients' quality of life. While there might be difficulties finding a measure of the extent of some of this improvement, as it can vary across individuals, multidisciplinary teams have the means to provide evidence of quality of life improvement based on observations the make in their daily interaction with patients and the knowledge acquired from pre- and post-surgery follow-ups. We are aware that a lot of work still needs to be done in this field, but more and more we find evidence of the surgery's benefits to patients. This evidence comes as an outcome of putting together multidisciplinary teams to closely monitor patients through the entire treatment and from continually perfecting techniques based on new data. It is important that we persist with this type of treatment as it has been shown again and again to be very successful in the treatment of morbid obesity.

Ronaldo Mafia CUENCA*

\section{REFERENCES}

1. Costa RC, Yamaguchi N, Santo MA, Riccioppo D, Pinto Jr PE. Outcomes on quality of life, weight loss, and comorbidities after Roux-en-Y gastric bypass. Arq Gastroenterol. 2014,51(3):165-70.

2. Andrade-Silva SG, Caranti DA, Sallet JA, Leal LPFF, Leal AJF, Dâmaso AR. Age and gender may influence the results of Roux-en-Y gastric bypass? Metabolic syndrome parameters. Arq Gastroenterol. 2014,51(3):171-9. 